\title{
Wireless Sensor Networks-based Solutions for Cattle Health Monitoring: A Survey
}

\author{
Mohamad Gameil \\ Electrical Engineering and Information Technology, RUR University Bochum, Germany
}

Tarek Gaber

Faculty of Computers and Informatics, Suez Canal University, Egypt

School of Computing, Science and Engineering, University of Salford, UK

Scientific Research Group in Egypt, (SRGE), Cairo, Egypt

Email: t.m.a.gaber@salford.ac.uk

\begin{abstract}
Wireless Sensor Networks (WSN) are nowadays becoming an active research in different fields. Precise irrigation, agriculture, earthquake, fire monitoring in forests and animal health monitoring are few applications of WSN. Animal health monitoring systems (AHMS) are usually used to monitor physiological parameters such as rumination, heart rate, and body temperature. Traditional methods to monitor animal health such as (traditional surveillance, single observation, and simple tabular and graphic techniques) are not efficient to achieve high performance in the large herds' management systems. These methods can only provide partial information and introduce a large cost in staffing and physical hardware. Thus, it is of important need to overcome a foresaid draw-back by using alternative low cost, low power consumption sensor nodes, and providing real-time communications at a sensible hardware cost. The objectives of this paper are: reviewing existing WSN solutions for cattle health monitoring models and determining the requirements needed for building an effective WSN model suitable for cattle health monitoring and detect animal diseases. From this review, requirements of the effective WSN-based solution for cattle health monitoring were suggested.
\end{abstract}

KEYWORDS: Wireless sensor networks, Zigbee, UART, rumination, cattle monitoring, animal health monitoring

\section{Introduction}

Humans rely on animals for food, fiber, labor and companionship. Thus, it is very crucial to keep these animals healthy and productive. According to the FAO, the world cattle population is estimated to be about 1.5 billion head. Hence cattle management becomes difficult by increasing the number of cow. In large herds, infection with enteric pathogens such as (E. coli) or Salmonella, and foot-and-mouth disease is common and associated with poor performance and animal welfare, as well as expensive treatment costs [1] [18].

These diseases can spread and infect other animals as well as humans. For these reasons, a system is needed to be in place for continuously monitoring the animal health. Technology is already part of modern farming and is playing an increasing role as more advanced systems and tools become available [1] [15] [19]. The new concepts and advancement in the technologies nowadays are Internet of Things (IoT). The main idea of IoT is getting real world objects connected with each other forming Wireless Sensor Network (WSN) which would help to control and prevent the eruption of diseases at large scale of cattle management. With the 
use of sensor, application on mobile phones and the transfer of useful data generated by the system will make it easy to use it [2].

Usually, there are two methods of monitoring animal health: indirect and direct contact (noninvasive) method. In the indirect method:

- Traditional methods (traditional surveillance / single observation) is the practices of disease reporting. Traditionally, this have been based on observation of activity with the naked eye.

- Simple tabular and Graphic techniques that analyze surveillance data, compare current data with some "expected" value and identify how these differ.

In the direct contact method (invasive / Information Technology Methods) of animal health monitoring, there are three methods.

- Video Magnification, is the act of making something look larger than it is, the act of magnifying something or the larger appearance of an object when it is seen through a microscope, telescope, etc. Video Magnification Disease revealing invisible changes in the world allowed you to see subtle changes that cannot be seen with the naked eye like respiratory motion, human pulses to extract heart rate, see invisible (tiny) motion and hear silent sounds [13].

- Location tracking using Image Processing Based on video footage from multiple cameras located in and around a pen, which houses the animals, to extract their location and determine their activity [14].

- Wireless sensor networks (WSN) is spatially distributed, collection of sensor nodes for the purpose of monitoring physical or environmental conditions, such as temperature, sound, pressure, Earthquake and Fire prediction etc, and to cooperatively pass their data through the network to a main location. WSN consists of distributed wireless enabled devices that have the ability to handle a variety of electronic sensors. Each node of the WSN called a mote and is accompanied with one or more sensors in addition to a microcontroller, wireless transceiver, and energy source $[3,16]$.

Wireless Sensor Networks are found to be more advantageous over traditional systems as the WSNs-based systems are founded on embedded construction and distributed nature and they are low cost, low power consumption, mesh networking scheme and inherit nature of RF communication transmission of data from one point to another among nodes in a mesh based topology network takes less energy. WSN has a better coverage than centralized traditional sensing technology [3]. WSNs-based solutions could keep quality of indoor environment that is very important for animal health and welfare which ultimately impacts productivity and quality

[17].

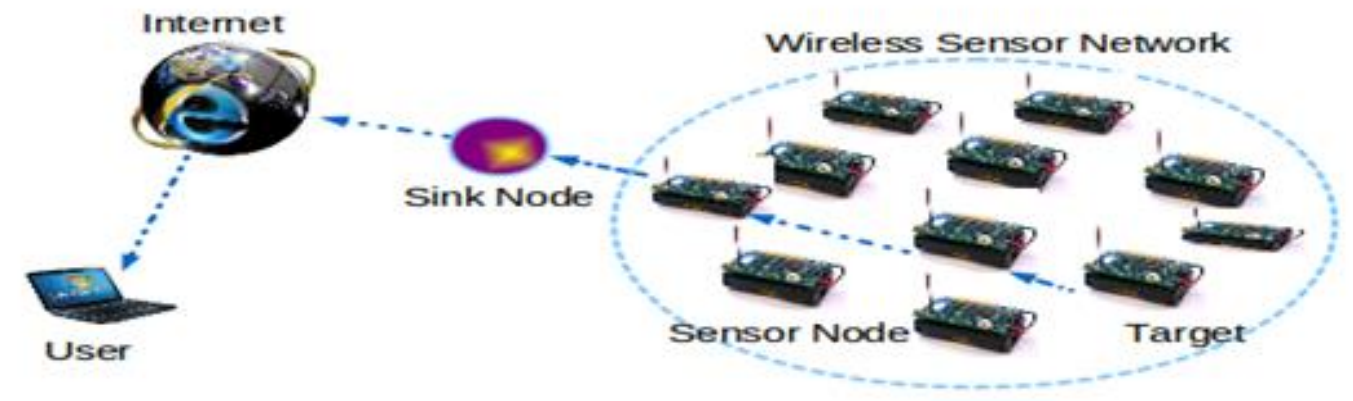


Fig.1 Collection of sensor nodes for the purpose of monitoring physical or environmental conditions, such as temperature, sound, pressure [3]

Two main standard technologies are usually used in WSN: ZigBee and Bluetooth. Both of these technologies operate within the Industrial Scientific and Medical (ISM) band of 2.4GHz. This band leads to license-free operations and huge spectrum allocation for compatibility. It is also possible to create a WSN using Wi-Fi (IEEE 802.11) which has high power consumption $[3]$.

The objectives of this paper are reviewing existing WSN solutions for cattle health monitoring models and determining the requirements needed for building an effective WSN model suitable for cattle health monitoring and detect animal diseases.

The rest of this paper is organized as the follows: Sect. 2 presents the related work; Sect. 3 conducts a comparison among some of the existing WSN models. Finally, Sect. 4 concludes the work done on this paper.

\section{Related Work}

RFID technology is among different electronic means for monitoring animal/cattle health. In RFID, tags or collars were placed on the neck and microphone is incorporated and sounds are analyzed through a complex algorithm inside the tag. However, in many cases monitoring body temperature becomes important. Many new technologies have been introduced to measure body temperature of cattle at various locations including ear, rectum, reticulum-rumen, skin and milk. Most of the existing AHMS models make their decision based on the use of Wi-Fi modules such as Zigbee, Bluetooth or UART and the use of GPS modules such as in ZebraNet or LynxNet modules. Therefore we classified the literature into the following two classes: Zigbee and GPS based animal health solutions.

\subsection{Zigbee-based Animal Health Solutions}

Zigbee communication has been used of the development of Animal health monitoring system (AHMS) as it is an energy efficient, high accuracy, self-configuring, and low cost communication technology. Zigbee communication has well-known applications such as environment monitoring, viginet (military), smart farms, smart building, telemedicine services, and other industrial applications. Zigbee module working on the $2.4 \mathrm{GHz}$ band, but data transmits and receives serially through UART. Zigbee module has configured through X-CTU software [1].

A. Kumar et al. [1] identified and addressed the problem of the continuous rise in air temperature in the troposphere and the variations in temperature that has harmful effect on animal's health leading to diseases such as foot-and-mouth disease and swine fever- these diseases can spread and infect other animals as well as humans. To address these problems, A. Kumar et al. [1] proposed a prototype tele monitoring system consists of sensing unit and receiving unit with $\mathrm{PC}$ which reported the animal health monitoring system with a capability to monitor heart rate, body temperature, and rumination with surrounding temperature and humidity. The sensing unit is consisting of sensor, processor, and ZigBee module. Sensors is used to measure parameters that have been used for different animal species health monitoring. The sensed data of the developed sensor are sent to a host computer through ZigBee module. 
The values of a foresaid parameters can be displayed on the GUI PC. The design of AHM system is a scalable device.

E.S. Nadimi et al. [2] proposed a system integrating the control of all the deployed systems in a single system. The central system (Base Station) is the heart of this system as it is responsible for communications between nodes and central server and database management as well as communication with the outer world, Experiment was carried out at Island in Denmark over 5 days with eleven sheep for $9 \mathrm{~h}$ per day. The node on the collar and the collar itself were fixed to prevent them from sliding to the right or left. Each sheep in the flock had a wireless sensor measuring and transmitting the head movement acceleration measurements with a sampling rate of $1 \mathrm{~Hz}$. The performance of the handshaking communication protocol and the successful use of acknowledgment messages used to enhance communication reliability. A $2.4-\mathrm{GHz}$ ZigBee-based mobile ad hoc wireless sensor network (MANET) aiming to monitor animal behavior parameters (head movements of each individual sheep in a herd) was successfully designed and established. The deployment of two relay nodes enhanced the network connectivity, and the multi-hop communication and handshaking protocol among the wireless nodes resulted in high communication reliability and low energy consumption.

Leena et al. [4] proposed a solution consists of four sections: Raspberry Pi, Accelerometer module, Temperature and humidity sensor modules. This AHMS detects the animal parameters such as rumination, body temperature along with surrounding temperature and humidity. Zigbee protocol is used for data transmission and reception. Raspberry Pi is used as web server and only authorized persons can access the collected data. Raspberry Pi is a basic low cost computer on a single-board. It uses Linux-kernel-based operating systems. Model B+ was upgraded version of Model B which includes an improved power circuitry for attaching high powered USB devices, and switching regulators that can be used to reduce power consumption.

Sonia et al. [6] proposed and implemented a disease forecasting system for pigs using a received signal through ZigBee-based wireless network using a 3-axis acceleration sensor to detect illness at an early stage by monitoring movement of experimentally infected weaned piglet. The movement of infected piglets was altered, and the acceleration sensor could be successfully employed for monitoring pig activity. Accelerometers are sensors that can be used as motion detectors as well as for body position and posture sensing. The overall objective was to investigate to what extent physical activity/movement changes in response to oral infection with S.enteritidis and E.coli, and to establish whether or not monitored behavior altered due to infection can be used as an early sign of pending disease induced by inoculated bacteria.

Myeong et al. [7] proposed an effective livestock monitoring system (LMS) using biosensors for cattle health monitoring systems. The monitoring system aims to collect biometric data directly associated with the diseases from an individual entity and prevent them from occurring or spreading. Zigbee module is employed for transmitting the collected biometric data to the forecasting system on WSN. The validity of the system was verified by comparing the results measured by a commercial ECG equipment for cattle to those of LMS in terms of the heartbeat and the breath rate.

\subsection{GPS-based Animal Health Solutions}

In this section, we will review two main solution based on GPS technology for animal health problems. There are two main solutions: ZebraNet and LynxNet.

\subsubsection{ZebraNet Module}


In the ZebraNet-based solutions, GPS devices are mounted on the Zebra to routinely exchange position data with all other devices that fall within their transmission range. If sufficient memory space is available, a user could then download historical position data of multiple animals by approaching a single zebra. A number of solutions based on the ZebraNet model is discussed as follow.

Kae et al. [8] have noticed a number of major animal disease outbreaks in the UK which the farming industry is an important sector of its economy. The two most significant incidents were the BSE and FMD outbreak where 4.5 million cows were burnt and over 4 million were killed to stop the spread of these diseases. ZebraNet model was used to track and monitor the health condition of individual cattle activity. However, this model is based on store and forward approach which is not efficient to achieve high performance. So, there is a need for an alternative low cost and low power consumption sensor nodes to support a real-time health monitoring application. To achieve this aim, a particular routing protocol is presented to assist multi hop connectivity that skips the time spent in creating and maintaining plain routing path that led to shorter packet delay.

To address the above problem, Tsung et al. [9] suggested a new routing protocol to address the connectivity problem between collars which would lead to an unstable routing path and resulting in increased packet delay. They proposed an Implicit Routing Protocol (IRP) consisting of two phases: configuration and data forwarding. In the configuration phase, the BS periodically send a TIER message throughout entire network contains a BS's ID field, and a hop count field. In the data forwarding phase, if the collar wants to report its measured data back to the base station, it will create a packet containing its current TIER ID and measurement data. This packet is then broadcasted to its vicinity which has a smaller TIER ID. This collar, after acknowledging to the source collar, will broadcast the received packet. This forwarding rule will then be repeated until the data reaches at the BS. Thus, the IRP protocol can reduce the impact of mobility under varying "Off" probability, and number of sensor node.

Dukki et al. [10] proposed a cow monitoring system consisting of real-time monitoring device, environment information device, activity parameter device and GPS device. Monitoring control middle ware is video control module, environment control module, monitoring setting module, location awareness control module and activity calculation module. Also, there are monitoring server system that can creates event for cow activity.

\subsubsection{LynxNet Module}

LynxNet system is based on tracking collars, built around T Mote Mini sensor nodes, sensors, GPS and $433 \mathrm{MHz}$ radio, and stationary base stations, placed at the locations that are visited frequently by the animals. This system is quite similar to ZebraNet but Lynx animal is smaller than a zebra so the latter requires more compact and lightweight solution. Reinholds et al. [11] proposed LynxNet system with extended sensing modality and multi hop delay tolerant communication approach to track Eurasian lynx migration in Latvian forests. The challenge is to achieve long-term operation with a single set of batteries. LynxNet nodes are producing two types of packets. The first type contains GPS location and fix quality information, temperature, relative humidity and amount of ambient light. In this type, one packet is formed once every hour. The second type of packets contains data from 3D accelerometer and 2D gyroscope that can be used to calculate motion vector. Every 5 minutes, 5 samples of data are gathered, stored 
in 5 packets to help with the lynx activity classification. These collected data are then analyzed to monitor the lynx health.

Similar to LynxNet-based solution, L. A. González et al. [12] proposed a method to identify the value of monitoring technologies of cattle grazing tropical pastures. To capture LW of three groups of 20 animals in an experiment of 341 days length, three remote weighing systems were set up at the water troughs. These collected LW data and data from monitored collars, sufficient detail in real-time making can give a valuable insight for early management interventions and right decisions. This consequently would result in an increased production, animal welfare and environmental stewardship. Observations of the data recorded by all three weighing stations throughout the 341 days of the experiment were 41824 observations. $35.8 \%$ not success rate of observations between missing EID number and outliers with LW records, i.e. $64.2 \%$ success rate.

\section{Comparison of WSN Solutions for Cattle Monitoring}

From the reviewed solutions above, we can determine the requirements of the effective WSNbased solution for cattle health monitoring:

- Wireless communication module: the device that collect data from different sensors, transmit the biometric data through sink module to the forecasting system.

- Mobile Sensors: measure bio-signals of cattle such as the heartbeat, the breath rate, body temperature, rumination, surrounding temperature, humidity and the momentum.

- Immobile Sensors: environmental fixed sensors such as (thermal camera, video camera, thermistor for surrounding temperature and humidity).

- Energy Consumption: the amount of energy consumed during the system lifetime.

- Cost: the amount of money that has to be paid or given up in order to get the devices.

- Real Experiment / Simulation: is the researchers implemented, simulate their project or just a proposal and how is the accuracy of the result.

- Addressing Security: the prevention of unauthorized access or damage to the forecasting system by applying special polices or protocols.

- Web/mobile system: the ability of controlling and monitoring system remotely.

- Future Enhancement: the future improvements that make the system agreeable.

\subsection{ZigBee-based Solutions}

- Symbol Y: Means that model did achieve this probability.

- Symbol X: Means that model did not achieve this probability.

From Table 1, it can be noticed that the ZigBee module is very helpful device of inexpensive health care of cattle management. It is an energy efficient, high accuracy, self-configuring, and low cost communication technology and no one uses fixed sensors.

Table 1: Comparison among ZigBee-based Solutions

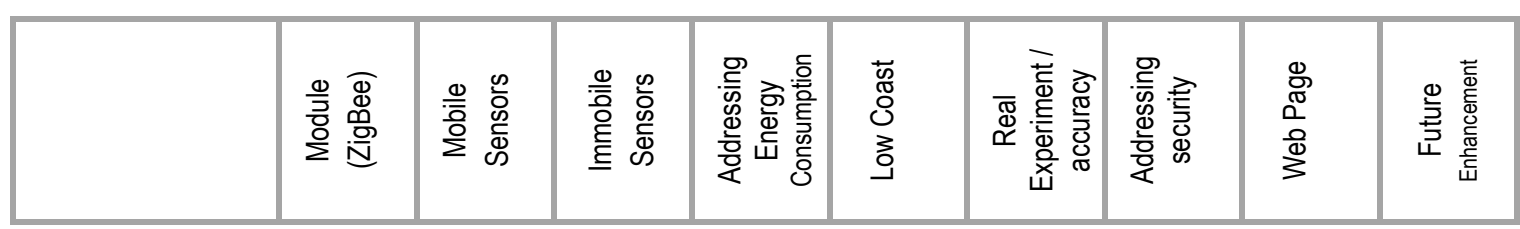




\begin{tabular}{|l|c|c|c|c|c|c|c|c|c|}
\hline $\begin{array}{l}\text { A. Kumar et } \\
\text { al. [1] }\end{array}$ & $\mathbf{Y}$ & $\mathbf{Y}$ & $\mathbf{X}$ & $\mathbf{Y}$ & $\mathbf{Y}$ & $\mathbf{X}$ & $\mathbf{X}$ & $\mathbf{X}$ & $\mathbf{Y}$ \\
\hline $\begin{array}{l}\text { E.S. Nadimi et } \\
\text { al. [2] }\end{array}$ & $\mathbf{Y}$ & $\mathbf{Y}$ & $\mathbf{X}$ & $\mathbf{Y}$ & $\mathbf{X}$ & $\mathbf{Y}$ & $\mathbf{Y}$ & $\mathbf{Y}$ & $\mathbf{Y}$ \\
\hline $\begin{array}{l}\text { Leena et al. } \\
\text { [4] }\end{array}$ & $\mathbf{Y}$ & $\mathbf{Y}$ & $\mathbf{X}$ & $\mathbf{X}$ & $\mathbf{Y}$ & $\begin{array}{c}\text { X } \\
\text { simulation }\end{array}$ & $\mathbf{X}$ & $\mathbf{Y}$ & $\mathbf{Y}$ \\
\hline Sonia et al. [6] & $\mathbf{Y}$ & $\mathbf{Y}$ & $\mathbf{X}$ & $\mathbf{X}$ & $\mathbf{Y}$ & $\mathbf{Y}$ & $\mathbf{X}$ & $\mathbf{X}$ & $\mathbf{X}$ \\
\hline $\begin{array}{l}\text { Myeong et al. } \\
\text { [7] }\end{array}$ & $\mathbf{Y}$ & $\mathbf{Y}$ & $\mathbf{X}$ & $\mathbf{Y}$ & $\mathbf{Y}$ & $\mathbf{Y}$ & $\mathbf{X}$ & $\mathbf{Y}$ & $\mathbf{X}$ \\
\hline
\end{tabular}

\subsection{GPS-based Solutions}

Various researchers use ZebraNet to track and monitor the health condition of individual animal activity. GPS position and Zebra Net, both schemes are based on store and forward approach. LynxNet system with extended sensing modality and multi hop delay tolerant communication approach able to achieve long-term operation. Table 2 shows that ZebraNet module is not efficient to achieve high performance.

Table 2: Comparison among GPS-based Solutions

\begin{tabular}{|c|c|c|c|c|c|c|c|c|c|c|}
\hline & \multicolumn{2}{|c|}{ Module } & \multirow{2}{*}{ 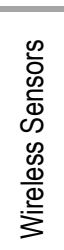 } & \multirow{2}{*}{ 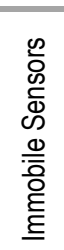 } & \multirow[b]{2}{*}{ 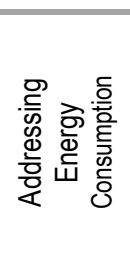 } & \multirow[b]{2}{*}{$\begin{array}{l}\text { 苋 } \\
0 \\
0 \\
3 \\
3\end{array}$} & \multirow{2}{*}{ 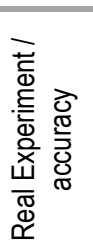 } & \multirow[b]{2}{*}{ 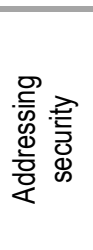 } & \multirow[b]{2}{*}{ 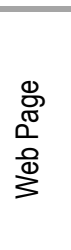 } & \multirow{2}{*}{ 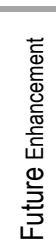 } \\
\hline & 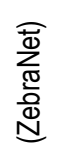 & 离 & & & & & & & & \\
\hline Kae et al. [8] & Y & $\mathbf{X}$ & $\mathbf{Y}$ & $\mathbf{X}$ & Y & Y & $\mathbf{X}$ & $\mathbf{X}$ & $\mathbf{X}$ & $\mathbf{X}$ \\
\hline $\begin{array}{l}\text { Tsung et al. } \\
\text { [9] }\end{array}$ & $\mathbf{Y}$ & $\mathbf{X}$ & $\mathbf{Y}$ & $\mathbf{X}$ & $\mathbf{Y}$ & $\mathbf{Y}$ & $\mathbf{X}$ & $\mathbf{X}$ & $\mathbf{X}$ & $\mathbf{X}$ \\
\hline $\begin{array}{l}\text { Dukki et al. } \\
\text { [10] }\end{array}$ & $\mathbf{Y}$ & $\mathbf{X}$ & $\mathbf{Y}$ & $\mathbf{Y}$ & $\mathbf{X}$ & $\mathbf{X}$ & $\mathbf{X}$ & $\mathbf{X}$ & $\mathbf{X}$ & $\mathbf{Y}$ \\
\hline $\begin{array}{l}\text { Reinholds et } \\
\text { al. [11] }\end{array}$ & $\mathbf{X}$ & $\mathbf{Y}$ & $\mathbf{Y}$ & $\mathbf{X}$ & $\mathbf{Y}$ & $\mathbf{X}$ & $\mathbf{Y}$ & $\mathbf{X}$ & $\mathbf{X}$ & $\mathbf{Y}$ \\
\hline $\begin{array}{l}\text { L. A. } \\
\text { González et } \\
\text { al. [12] }\end{array}$ & $\mathbf{X}$ & $\mathbf{Y}$ & $\mathbf{Y}$ & $\mathbf{Y}$ & $\mathbf{X}$ & $\mathbf{X}$ & $\mathbf{Y}$ & $\mathbf{X}$ & $\mathbf{Y}$ & $\mathbf{X}$ \\
\hline
\end{tabular}

\section{Conclusion}

In this paper, we discussed the importance of animals in our life and why we need to keep the animals healthy and productive. Also, we highlighted the traditional methods used for monitoring cattle and how these methods are not efficient to achieve high performance in the large herds management systems. Therefore, we surveyed the solutions that are proposed to address the limitation of traditional methods through Wireless Sensor Networks (WSN) that found to be more advantageous over traditional sensing technology, GPS since the WSNsbased systems are low cost, low power consumption sensor nodes, and providing real-time communications at a sensible hardware cost. Also, a comparison among the proposed solutions 
were conducted based identified requirements for effective cattle health monitoring system based on WSN. In the future, the security problems would be identified and addressed. This is important as the collected and analyzed should be reliable (not tampered with during its transmission from the sensor nodes to the sink node). Otherwise, the decisions based on these data would not be effective. So security service such as integrity, availability and authentication should be addressed in cattle health monitoring systems.

\section{References}

1. Kumar, A. and Hancke, G.P., 2014. A zigbee-based animal health monitoring system. IEEE sensors Journal, 15(1), pp.610-617.

2. Nadimi, E.S., Jørgensen, R.N., Blanes-Vidal, V. and Christensen, S., 2012. Monitoring and classifying animal behavior using ZigBee-based mobile ad hoc wireless sensor networks and artificial neural networks. Computers and Electronics in Agriculture, 82, pp.44-54.

3. Neethirajan, S., 2017. Recent advances in wearable sensors for animal health management. Sensing and Bio-Sensing Research, 12, pp.15-29.

4. Narayan, L., Muthumanickam, D.T. and Nagappan, D.A., 2015. Animal Health Monitoring System using Raspberry Pi and Wireless Sensor. International Journal of Scientific Research and Education (IJSRE), 3(5).

5. Taylor, S. M.; and Andrews, A.H. (1992)" Endoparasites and Ectoparasites In:Bovine Medicine Diseases and Husbandry of Cattle" 1st Edit, (Andrews et al., eds), Oxford, Blackwell Scientific Publications London, EdinBurgh, Boston.

6. Ahmed, S.T., Mun, H.S., Islam, M.M., Yoe, H. and Yang, C.J., 2016. Monitoring activity for recognition of illness in experimentally infected weaned piglets using received signal strength indication ZigBee-based wireless acceleration sensor. Asian-Australasian journal of animal sciences, 29(1), p.149.

7. Park, M.C. and Ha, O.K., 2015. Development of effective cattle health monitoring system based on biosensors. Adv. Sci. Tech, 117, pp.180-185.

8. Kwong, K.H., Wu, T.T., Goh, H.G., Stephen, B., Gilroy, M., Michie, C. and Andonovic, I., 2009. Wireless sensor networks in agriculture: Cattle monitoring for farming industries. Piers Online, 5(1), pp.31-35.

9. Wu, T., Swee Keow Goo, Kae Hsiang Kwong, Craig Michie, and Ivan Andonovic. "Wireless sensor network for cattle monitoring system." (2009): 173-176. Available at https://strathprints.strath.ac.uk/14614/ (Last accessed 25/07/2019)

10. Dukki Kim1, Hyun Yoe, "Cow Monitoring System based on Event using Wireless Sensor Network", Advanced Science and Technology Letters Vol.95 (CIA 2015), pp.173-176.

11. Reinholds Zviedris, Atis Elsts, Girts Strazdins, Artis Mednis.: LynxNet: Wild Animal Monitoring Using Sensor Networks. REALWSN 2010, LNCS 6511, pp. 170-173, 2010. CSpringer-Verlag Berlin Heidelberg 2010.

12. González, L.A., Bishop-Hurley, G., Henry, D. and Charmley, E., 2014. Wireless sensor networks to study, monitor and manage cattle in grazing systems. Animal Production Science, 54(10), pp.1687-1693.

13. Takeda, S., Akagi, Y., Okami, K., Isogai, M. and Kimata, H., 2019. Video Magnification in the Wild Using Fractional Anisotropy in Temporal Distribution. In Proceedings of the IEEE Conference on Computer Vision and Pattern Recognition (pp. 1614-1622)..

14. Dao, T.K., Le, T.L., Harle, D., Murray, P., Tachtatzis, C., Marshall, S., Michie, C. and Andonovic, I., 2015, August. Automatic cattle location tracking using image processing. In 2015 23rd European Signal Processing Conference (EUSIPCO) (pp. 2636-2640). IEEE. 
15. Molapo, N.A., Malekian, R. and Nair, L., 2019. Real-Time Livestock Tracking System with Integration of Sensors and Beacon Navigation. Wireless Personal Communications, 104(2), pp.853-879.

16. Behera, T.M., Mohapatra, S.K., Samal, U.C. and Khan, M.S., 2019. Hybrid heterogeneous routing scheme for improved network performance in WSNs for animal tracking. Internet of Things, 6, p.100047.

17. Priya, M.K. and Jayaram, B.G., 2019. WSN-Based Electronic Livestock of Dairy Cattle and Physical Parameters Monitoring. In Emerging Research in Electronics, Computer Science and Technology (pp. 37-45). Springer, Singapore.

18. Kiani, F., 2018. Animal behavior management by energy-efficient wireless sensor networks. Computers and electronics in agriculture, 151, pp.478-484.

19. Moreno-Moreno, C.D., Brox-Jiménez, M., Gersnoviez-Milla, A.A., Márquez-Moyano, M., Ortiz-López, M.A. and Quiles-Latorre, F.J., 2018. Wireless Sensor Network for Sustainable Agriculture. Multidisciplinary Digital Publishing Institute Proceedings, 2(20), p.1302. 